\title{
Editorial
}

\section{Asia Tsunami: putting hard learned lessons into action}

\begin{abstract}
A lethal tidal wave devastated several countries in Asia including Sri Lanka on the $26^{\text {th }}$ of December 2005. The county was taken by surprise causing unprecedented loss of human lives and property. It created a colossal job for the disaster management plan of the country. Sri Lanka has not experienced a natural disaster of this magnitude and rapidity in the recent history. The disaster management plan never seemed to be geared for an operation of this magnitude.
\end{abstract}

Media and the public took over the task of providing emergency relief measures. How well this job was done has never been assessed and people were forced to rely on media competing with each other for information and co-ordination. However, if not for the reflex response from the public and media there would have been much more post Tsunami deaths and suffering.

Apart from the reflex response from the general public which kept those who survived fed and cared for, public health infrastructure provided much needed back bone to provide relief measures, health and medical care. Divisional Directors of Health, Medical Officers of Health $(\mathrm{MOH})$ and their staff were working round the clock with local government officials in setting up camps and coordinating supplies and care providers. Some of the staff members including MOHs had been fortunate enough to survive having been caught in the waves though lot of damage had happened to their property. Leaving all these behind they were back at work helping others. This commitment has passed largely unnoticed. Focus of media was mostly on the distribution of supplies and on the foreign relief teams. At least the College of Community Physicians should acknowledge and appreciate the courage and dedication of $\mathrm{MOHs}$ and their staff.

It is worth noting that there were no post disaster outbreaks of communicable diseases in Sri Lanka. It is an achievement in itself. Those who were largely responsible were the $\mathrm{MOHs}$ and their teams assisted by many others. Many MOHs were very receptive to help when offered and tried to get the maximum use of what has been offered.

What was most notable was the ineffective coordination at national level. Those who offered to help and contacted officials at central level were made to feel whether a disaster management plan ever existed in this country. Lot of people who offered help felt unwelcome and were sent from pillar to post. There was no proper mechanism of coordinating volunteer groups and supplies and sending those to appropriate places. This was a failure on the part of the disaster management plan and ultimately the government

All natural disasters cannot be predicted. It is also now known that a new micro tectonic plate has been created three hundred kilometers south of Sri Lanka. It has already caused tremors amounting to five in Richter scale and is likely to cause earth tremors and tsunamis in future as well. If these will really happen Sri Lanka will be in a very dangerous proximity to those seismic foci and epicenters (1).

We need to learn from this lesson. I suggest the following measures to be considered in future disaster management:

1. A well structured disaster management unit should be created. A protocol should be developed with suitable persons to head different aspects like:

- Immediate relief and life saving with help from armed forces and police.

- Rapid supply of immediate needs like cooked food, water, clothes and shelter.

- Coordination of humanitarian supplies and human resource support

- Health and medical care management

- media coordination

- situation monitoring and data gathering

- long term management (rehabilitation and rebuilding)

- A natural disaster warning system

2. Frequent disaster management drills should be performed to keep the preparedness of the team at an optimum level.

3. Those who dedicated themselves to work should be rewarded.

4. Government and non governmental institutions should be directed to train their workers to manage disaster situations in local areas and to help each other in such situations. Expert guidelines (2) should be made available to all of them. Organizations like police need to be trained to be deploy in life saving and 
immediate relief work. Had that been done earlier, few more lives could have been saved at least in the ill fated train at Telwatta

Establishing and running of the disaster management unit should be directly under a responsible cabinet minister who should be held responsible for its operational success. This matter should be considered urgent and given utmost national importance.

\section{Reference:}

1. Karunaratne HKN. Will there be earthquakes in our country? Dinamina 2005 Feb. 08; sect.Sahashra 13 (col.2).

2. Karunathilake I, Editor. Guidelines for disaster management: a compilation of expert guidelines on providing health care, Colombo: Faculty of Medicine, University of Colombo; 2005.

Dr. Wasantha Gunathunga

Editor

February 2005 